\title{
Activated Human Eosinophils
}

\author{
Rossana C.N. Melo ${ }^{a, b}$ Peter F. Weller ${ }^{b}$ Ann M. Dvorak ${ }^{c}$ \\ a Laboratory of Cellular Biology, Department of Biology, Federal University of Juiz de Fora, UFJF, \\ Juiz de Fora, Brazil; Departments of ${ }^{\mathrm{b}}$ Medicine and ${ }^{\mathrm{C}}$ Pathology, Beth Israel Deaconess Medical Center, \\ Harvard Medical School, Boston, Mass., USA
}

Eosinophils are leukocytes which, when stimulated with cytokines or chemokines, become activated and release mediators stored in their dominant population of cytoplasmic granules (termed specific or secondary granules) [1]. The morphology of activated eosinophils is quite distinct. In a range of inflammatory and allergic disorders or upon physiological stimulation, activated eosinophils are generally seen as cells full of granules, but these structures are undergoing progressive losses of their contents with retention of granule outer membranes, a process known as 'piecemeal degranulation' [2, 3] (fig. 1a). As a result, a mixed population of intact (fig. 1a, asterisks) and enlarged, emptying (fig. 1a, arrows) granules is always visualized in cell sections [3]. Also, a decrease of specific granule numbers can occur [3]. A rare view of an activated eosinophil is shown in figure $1 \mathrm{~b}$ taken from a hypereosinophilic subject. While a single specific granule $(\mathrm{Gr})$ is seen in the cytoplasm in conjunction with an osmiophilic lipid body (LB), the cell surface shows elaborate projections indicative of activation. Vesiculotubular structures (fig. 1b, arrowheads), termed 'eosinophil sombrero vesicles' (EoSV) and recently associated with the eosinophil secretory pathway $[4,5]$ reside in the cytoplasm. This vesicle population is unique to eosinophils and allows the prompt identification of these cells by electron microscopy when specific granules are not present [3].

Shape changes are another hallmark of activated human eosinophils. In parallel with the morphological al-

terations of specific granules, eosinophils are able to change their morphology in response to different agonists. Figure 2 shows shape changes in eosinophils stimulated by eotaxin, a potent eosinophil activator.

\section{Acknowledgements}

Supported by NIH grants AI33372, AI20241, AI22571, HL70270. R.C.N.M. was supported by Conselho Nacional de Desenvolvimento Científico e Tecnológico (CNPq, Brazil).

References

Correspondence to: Dr. Ann M. Dvorak

Beth Israel Deaconess Medical Center

Department of Pathology, 330 Brookline Avenue

Boston, MA 02215 (USA)

Tel. +1 617667 3692, Fax +1 617667 2943, E-Mail advorak@bidmc.harvard.edu 


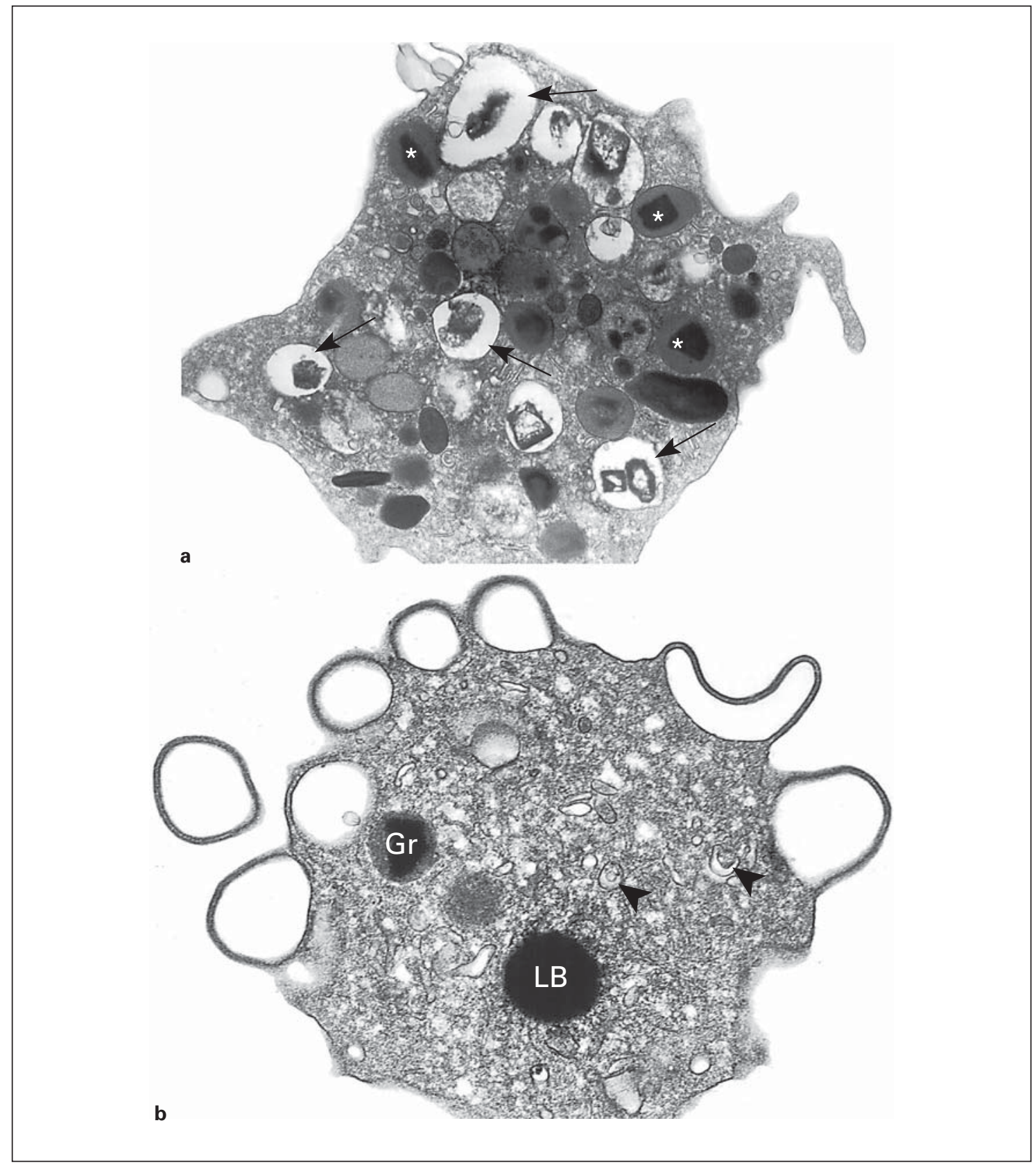

Fig. 1. Different views of activated eosinophils. a Eotaxin-stimulated eosinophils show piecemeal degranulation (arrows). Intact granules are indicated by asterisks. b Eosinophil from a hypereosinophilic subject. Arrowheads indicate eosinophil sombrero vesicles. $\mathrm{Gr}=$ Granule; $\mathrm{LB}=$ lipid body. $\mathbf{a} \times 20,000 . \mathbf{b} \times 25,000$. 


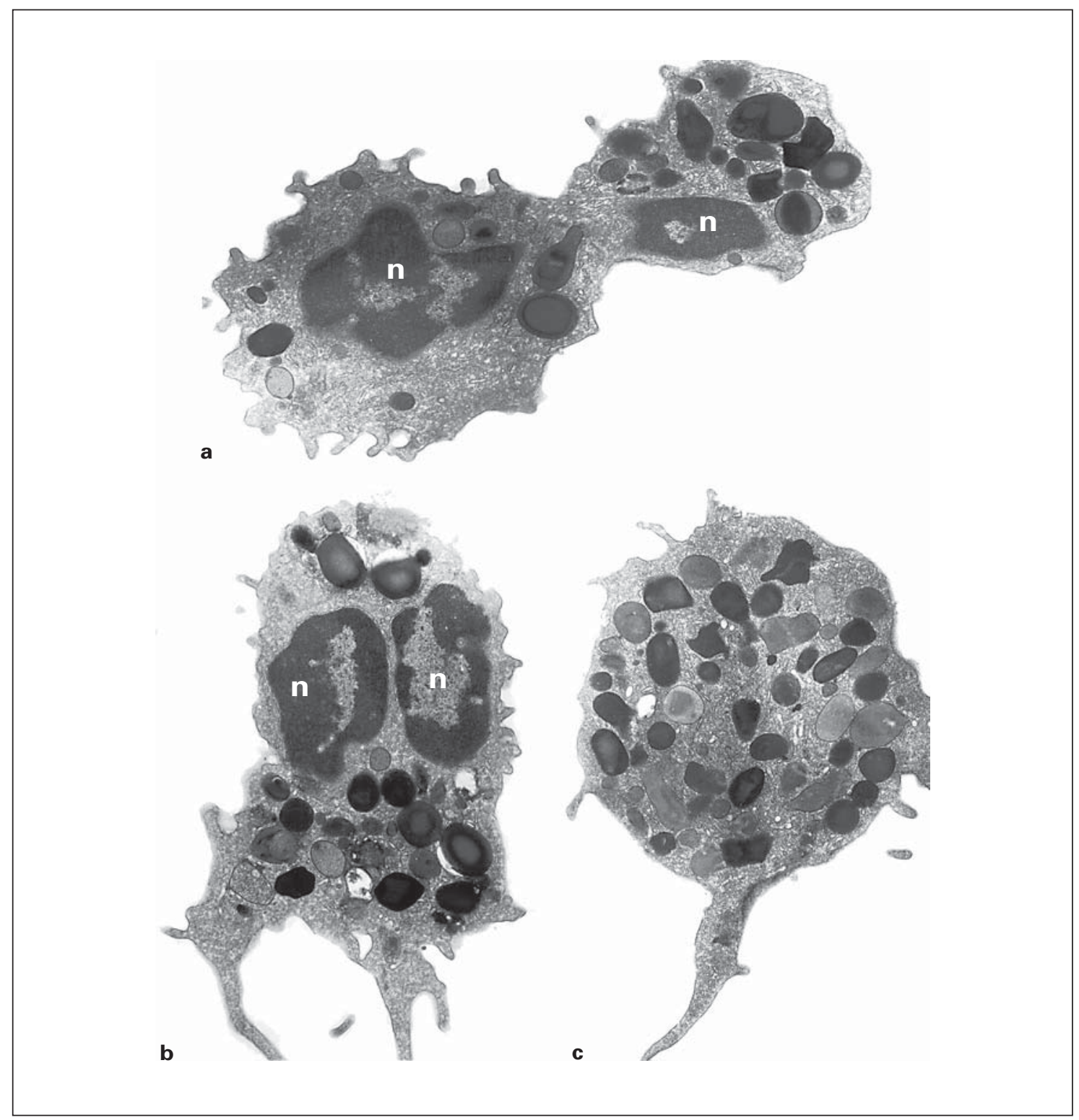

Fig. 2. Shape changes in eotaxin-activated eosinophils. a The eosinophil shows a narrow constriction at the midpoint of the cell. b The cell shows a broadened cytoplasmic area filled with granules and displaying broad surface processes. c Granule-free uropod is present. $\mathrm{n}=$ Nucleus. $\mathbf{a}-\mathbf{c} \times 18,000$. Eosinophils were isolated from the peripheral blood of healthy (fig. 1a, 2a, c) or hypereosinophilic (fig. 1b) donors by negative selection, stimulated (fig. 1a, 2a, c) or not (fig. 1b) with recombinant human eotaxin and prepared for transmission electron microscopy using standard methods. 